\title{
Minding the gap between clinical guidelines and real-life clinical work
}

\author{
Livia Balan-Moshe ${ }^{1,2} \cdot$ Doron Gothelf ${ }^{1,2,3}$
}

Published online: 1 April 2021

○) Springer-Verlag GmbH Germany, part of Springer Nature 2021

Today, we know that attention deficit/hyperactivity disorder (ADHD) is a lifelong disorder associated with impairments in multiple areas of functioning including school and learning, life skills, self-concept, social activities and risky behaviors [1]. The gold standard treatment for ADHD is multimodal, consisting both of medications, with stimulants being the first-line treatment, and psychosocial interventions [2-4]. Stimulants are very effective, resulting in substantial improvement of ADHD symptoms. In fact, stimulants are one of the most effective psychopharmacological therapies in psychiatry with an effect size of about 0.8 . Nevertheless, long-term studies have shown a gap between the high efficacy of stimulants found in clinical trials, as also reported in clinical guidelines, and the less favorable long-term outcome of treatment reported in real clinical practice [5].

In this issue, Mücke et al. [6] report on the adherence of physicians and therapists to guidelines for the assessment and treatment of children and adolescents with ADHD in Germany. They found a gap between the utopia as presented in guidelines and the clinical practice in naturalistic settings. Moreover, they found that physicians follow clinical guidelines during initial assessment better than during follow-up visits.

There are many challenges in the clinical treatment of ADHD, in the initiation, adherence, persistence, and optimization of treatment as we will describe below. Only about half of the children prescribed ADHD medications use the first prescription. Adherence to medications, defined in most studies as the number of days of medication supplied during a prespecified period, at the long run is very low. For

Livia Balan-Moshe

gothelf@tauex.tau.ac.il

1 The Child \& Adolescent Psychiatry Division, Edmond and Lily Safra Children's Hospital, Sheba Medical Center, 5262000 Tel Hashmoer, Israel

2 Sackler Faculty of Medicine, Tel Aviv University, Tel Aviv, Israel

3 Sagol School of Neuroscience, Tel Aviv University, Tel Aviv, Israel example, in the MTA study, only $7 \%$ of individuals with ADHD consistently used medications at least $50 \%$ of days, at all intervals, along 15 years of follow-up [5]. Persistence to medications in ADHD seems to be another challenge. It is defined as the duration of time in which medication treatment is continued and is measured from initiation of treatment to discontinuation. Persistence was reported to be relatively short for ADHD medications, with a mean duration of about 3-6 months [7].

Various factors affect medication initiation, persistence and long-term adherence. Some are not subjected to change, such as sociodemographic status and age at treatment. Yet, there are important modifiable factors that can substantially improve treatment success including psychoeducation on ADHD and its treatment, optimized dose titration and follow-up of side effects and their management. These modifiable factors are addressed in major clinical guidelines for the assessment of ADHD and its treatment [2-4].

Guidelines are periodically updated and describe the "gold standard" recommendations for assessment, treatment and long-term follow-up of children, adolescents and adults with ADHD. They all highlight the importance of psychoeducation as the first intervention. The guidelines for psychoeducation recommendations are extremely informative and include information about symptoms of ADHD and the effect of ADHD on different life domains, effective modes of communication with the child and effective behavioral management of everyday routines. Some guidelines, such as The Canadian ADHD Resource Alliance (CADDRA [2]), recommend the use of questionnaires for the assessment of ADHD symptoms and suggest the use of functional impairment rating scales at baseline assessment as well as at follow-up visits; whereas other guidelines, such as the NICE [3] and the AAP [4] mention the use of rating scales for ADHD symptoms assessment only as optional.

In this issue, Coghill et al. [1] demonstrate the heuristic value of including the well-established Weiss Functional Impairment Rating Scale-Parent Report as an outcome measure in phase 3 clinical trials of ADHD medications. This study shows that relying only on symptomatic scales 
misses important functional aspects of ADHD, which in many cases represent the cause and the motivation for initiating treatment and serve as a prognostic factor for later psychopathology [8]. In fact, an important facilitator for adherence to treatment in children with ADHD is the parents' understanding that ADHD affects many functional aspects and that medications improve not only class performance, but also those multiple domains of the child's functioning including behavior outside school and social functioning [7].

Coghill et al. [1] also showed that different drug classes have different effects on these functional areas. For example, only stimulants were significantly associated with reduced risky activities, while non-stimulant medications were associated with improvement in social functioning and none of the studied medications improved self-concept. The nonhomogenous effect of different drug classes on different functional domains makes an interesting case for personalization of medication based on functional impairment domains.

Although it is well accepted that broad clinical assessment that includes symptomatic and functional scales is important for optimizing ADHD treatment, the findings of Mücke et al. [6] are in line with the few other studies that assessed clinicians' adherence to clinical guidelines, showing that overall there is a low adherence of clinicians to clinical guidelines especially at follow-up visits [9] that exists also with other type of drugs used in the pediatric population [10]. We believe that the gap between clinical guidelines and their implementation represents the gap that exists between well-funded clinical trials and the time- and resource-limited reality of clinical practice. In the well-funded randomized controlled trials, patients are compensated for allocating ample time for research assessments and these assessments are conducted by funded research assistants, and physicians with protected time dedicated to thorough and structured assessment of patients. This enables using a comprehensive battery of assessment questionnaires in clinical trials. The physicians who are on the committees of clinical trials usually recommend these heavy batteries which are impossible to implement in the reality of time-limited assessment and follow-up visits, carried out without any research assistants available to administer the questionnaires and with limited time to provide the highly important but lengthy psychoeducation.

\section{Towards realistic clinical guidelines for the treatment of ADHD}

We believe that current guidelines should be adjusted so that the average clinical settings will be able to better adhere to guidelines recommendations. As mentioned above, assessment of both ADHD symptoms and functional domains are important for increasing adherence to treatment as well as optimizing its effect. Several studies have shown the significance of optimizing stimulants' dose, attained by frequent titration visits, to achieve stronger amelioration of ADHD symptoms [11], as well as the accompanying oppositional and aggressive symptoms in children [12]. Furthermore, low adherence to medications is sometimes due to patients' subjective feeling of ineffectiveness of medications [7]. These results emphasize the need of systematic symptoms and functional assessment for optimizing treatment using rating scales.

However, to be suitable for the average clinical setting, the recommended battery of questionnaires should be relatively short and efficient. The studies on adherence of physicians to clinical guidelines indicate that they use questionnaires in the initial assessment but tend not to do so at follow-up visits [6]. We, therefore, recommend to design the questionnaires-based follow-up assessment much more concise and focused. For example, only those items of ADHD symptoms and functioning with above threshold or high scores, as endorsed by the patients and their parents at baseline assessment, should be re-administered at follow-up. Those same items should also be presented to the physician in comparison to the same item reports in previous assessments, so change will be clearly apparent to both the physicians and patients. This is opposite to the need for blindness of both patients and physicians to previous visit scores required in clinical trials.

As for monitoring of side effects, the overlap between common comorbidities of ADHD and potential side effects includes sleep disorders, dysphoria, tic disorders and poor appetite. Therefore, it is important to screen those symptoms at baseline and at follow-up visits. If present at baseline, a symptom should be considered as a side effect only if it increased in severity from baseline to follow-up.

To further optimize the time at the clinic, patients and their parents should complete assessment questionnaires via their cellular phone while in the waiting room before entering the physician's room. Their responses should be processed and inserted automatically to the electronic medical record and presented to the clinician in simple graphs showing baseline vs. follow-up levels of symptoms and functional impairment, as well as reported side effects. To our knowledge, existing software programs that are able to manage such clinical data processing are scarce as most software, e.g., REDCap or Qualtrics, are designed for research and not for clinical purposes.

The waiting room should also be used for psychoeducation. Relevant data regarding ADHD symptoms, function and treatment should be offered for both the child and parents in electronic modalities, accessible and informative. The digital information may be personalized according 
to the patient's key domains of deficit and interest, as revealed by the questionnaires.

We believe that bridging the wide gap, which currently exists between the daily clinical setting and the rather utopic guidelines, is of utmost importance for improving the ability of clinicians to better adhere to ADHD clinical guidelines.

\section{References}

1. Coghill DR, Werner-Kiechle T, Farahbakhshian S et al (2020) Functional impairment outcomes in clinical trials of different ADHD medications: post hoc responder analyses and baseline subgroup analyses. Eur Child Adolesc Psychiatry. https://doi.org/ 10.1007/s00787-020-01586-5

2. Canadian ADHD Resource Alliance (CADDRA) (2018) Canadian ADHD Practice Guidelines, Fourth Edition, Toronto ON. https:// www.caddra.ca/wp-content/uploads/CADDRA-Guidelines-4thEdition_-Feb2018.pdf

3. National institute for Health and Care Excellence (2018) Guideline NG87 attention deficit hyperactivity disorder: diagnosis and management. https://www.nice.org.uk/guidance/ng87

4. Wolraich ML, Hagan JF, Allan C et al (2019) Clinical practice guideline for the diagnosis, evaluation, and treatment of attentiondeficit/hyperactivity disorder in children and adolescents. Pediatrics. https://doi.org/10.1542/peds.2019-2528

5. Swanson JM, Arnold LE, Molina BSG et al (2017) Young adult outcomes in the follow-up of the multimodal treatment study of attention-deficit/hyperactivity disorder: symptom persistence, source discrepancy, and height suppression. J Child Psychol Psychiatry Allied Discip 58:663-678. https://doi.org/10.1111/jcpp. 12684
6. Mücke K, Plück J, Steinhauser S et al (2020) Guideline adherence in German routine care of children and adolescents with ADHD: an observational study. Eur Child Adolesc Psychiatry. https://doi. org/10.1007/s00787-020-01559-8

7. Khan MU, Aslani P (2019) A review of factors influencing the three phases of medication adherence in people with attentiondeficit/hyperactivity disorder. J Child Adolesc Psychopharmacol 29:398-418. https://doi.org/10.1089/cap.2018.0153

8. Powell V, Riglin L, Hammerton G et al (2020) What explains the link between childhood ADHD and adolescent depression? Investigating the role of peer relationships and academic attainment. Eur Child Adolesc Psychiatry 29:1581-1591. https://doi.org/10. 1007/s00787-019-01463-w

9. Ellis LA, Blakely B, Hazell P et al (2021) Guideline adherence in the management of attention deficit hyperactivity disorder in children: an audit of selected medical records in three Australian states. PLoS ONE 16:e0245916. https://doi.org/10.1371/journal. pone. 0245916

10. Dinnissen M, Dietrich A, van der Molen JH et al (2020) Prescribing antipsychotics in child and adolescent psychiatry: guideline adherence. Eur Child Adolesc Psychiatry 29:1717-1727. https:// doi.org/10.1007/s00787-020-01488-6

11. Coghill D, Seth S (2015) Effective management of attentiondeficit/hyperactivity disorder (ADHD) through structured reassessment: The Dundee ADHD Clinical Care Pathway. Child Adolesc Psychiatry Ment Health 9:52-75

12. Blader JC, Pliszka SR, Kafantaris V et al (2021) Stepped treatment for attention-deficit/hyperactivity disorder and aggressive behavior: a randomized, controlled trial of adjunctive risperidone, divalproex sodium, or placebo after stimulant medication optimization. J Am Acad Child Adolesc Psychiatry 60:236-251. https:// doi.org/10.1016/j.jaac.2019.12.009 